\title{
Low Power FPGA Based Solar Charge Sensor Design Using Frequency Scaling
}

\author{
Puneet Tomar' ${ }^{1}$, Sheifali Gupta², Amanpreet Kaur², Sweety Dabbas³, \\ D M Akbar Hussain ${ }^{4}$
}

${ }^{1}$ South Asian University, New Delhi, India

${ }^{2}$ Chitkara University, Punjab, India

${ }^{3}$ Maharaja Surajmal Institute, New Delhi, India

${ }^{4}$ Aalborg University, Denmark

puneettomar91@gmail.com, sheifali.gupta@ chitkara.edu.in, amanpreet.kaur@chitkara.edu.in, sweety. dabas@gmail.com, akh@et.aau.dk,

\begin{abstract}
Resources of energy are degrading day by day the concept of energy saving is very important. Solar chargers are very most widely used devices which saves our energy resources. Use of Solar charges is now extremely increased. But the performance and effective output of these chargers depend upon how they are charged and discharged. Concept here is to use the solar Charger Sensor that regulates the charging and discharging process of Solar Chargers. These Solar charger Sensors should consume least power and hence make overall lifespan of these batteries to increase. Virtex6 Field Programmable Gate Array is used to Design the same Sensor for Solar Chargers for LVCMOS family. Frequency Scaling is used and reductions in Power consumed are observed.
\end{abstract}

Keywords- Energy Resources, Frequency Scaling, Solar Charger Sensor, Output, Discharging, Conservation

\section{INTRODUCTION}

Solar energy is best source for energy conservation concept and to use solar energy Solar Chargers are used. Solar chargers are commonly made up of number of cells which are connected together to store energy. The stored energy can be further used for many purposes like heating water or running electrical appliances and so on. The life span of solar chargers plays a very important role. If batteries are charged more than required as well as charging degrades below minimum level the performance will be affected. So, it is important for efficient output to charge them with utmost care. Solar Charge sensor can be used for this purpose. They sense the amount of voltage and stop and start charging the solar cells accordingly. We have done analyses on making these sensors low power and more precise. Frequency scaling is done for LVCMOS12, LVCMOS15, LVCMOS18 and LVCMOS 25. The frequencies used are from $0.01 \mathrm{GHz}$ to $100 \mathrm{GHz}$. Virtex6 Field Programmable array is used for implementing the design. The data is studied carefully and percentage changes for the factors that contribute to total power consumption of the sensor are calculated as shown in figure1, figure2, figure 3 and figure 4.

\section{RELATED WORK}

The concept of frequency scaling on FPGA is used by many researchers before for many Designs to minimize the power consumptions. LVCMOS based energy efficient charger [1] is analyzed before by using frequency scaling but the frequencies are different. The work is done for different LVCMOS Standards at different Frequencies used in our research. The frequencies used in related paper are $900 \mathrm{MHz}, 5 \mathrm{GHz}$ and $60 \mathrm{GHz}$ and Low voltage Complementary Metal Oxide used are LVCMOS15, LVCMOS18, LVCMOS25 and LVCMOS33. LVCMOS33 is not used by us in our paper and frequency range is also 
different. But in both the papers results are taken out from Xilinx tool. Some other papers that have somehow similar work as ours are Energy efficient processor design using frequency scaling [2], dynamic Frequency and voltage control for clock domain micro architecture [3], Efficient charging of capacitors for increasing lifespan of wireless sensor nodes [4] which works for energy conservation.

\section{DATA ANALYSIS AND INTERPRETATION}

\section{A. Result for LVCMOS12}

Table 1: Readings of I/Os, Leakage and Total Power for LVCMOS12 at frequency range from $0.01 \mathrm{GHz}$ to $100 \mathrm{GHz}$

\begin{tabular}{|l|l|l|l|l|l|}
\hline $\begin{array}{l}\text { Frequency } \\
\mathrm{GHz}\end{array}$ & 0.01 & 0.1 & 1 & 10 & 100 \\
\hline $\mathrm{I} / \mathrm{Os}$ & 0.000 & 0.001 & 0.007 & 0.065 & 0.653 \\
\hline Leakage & 0.710 & 0.710 & 0.711 & 0.715 & 0.760 \\
\hline Total Power & 0.710 & 0.712 & 0.731 & 0.921 & 2.809 \\
\hline
\end{tabular}

Readings noted in Table1 are analyzed and decrease in power consumption is noticed. The data can be analysed clearly from Figure 3 for LVCMOS12 and Percentage decrease are as follows. Decrease in I/O is $90.4 \%, 98.92 \%, 99.84 \%$ and $100 \%$ when frequency is reduced from $100 \mathrm{GHz}$ to $10 \mathrm{GHz}, 1 \mathrm{GHz}, 0.1 \mathrm{GHz}, 0.01 \mathrm{GHz}$ respectively. Decrease in Leakage is $5.92 \%, 6.44 \%, 6.57 \%$ and $6.57 \%$ when frequency is reduced from $100 \mathrm{GHz}$ to $10 \mathrm{GHz}, 1 \mathrm{GHz}$, $0.1 \mathrm{GHz}$, and $0.01 \mathrm{GHz}$ respectively. Decrease in Total Power is $67.2 \%, 73.97 \%, 74.65 \%$ and $74.72 \%$ when frequency is reduced from $100 \mathrm{GHz}$ to $10 \mathrm{GHz}, 1 \mathrm{GHz}, 0.1 \mathrm{GHz}$, and $0.01 \mathrm{GHz}$ respectively as shown in Fig. 1.

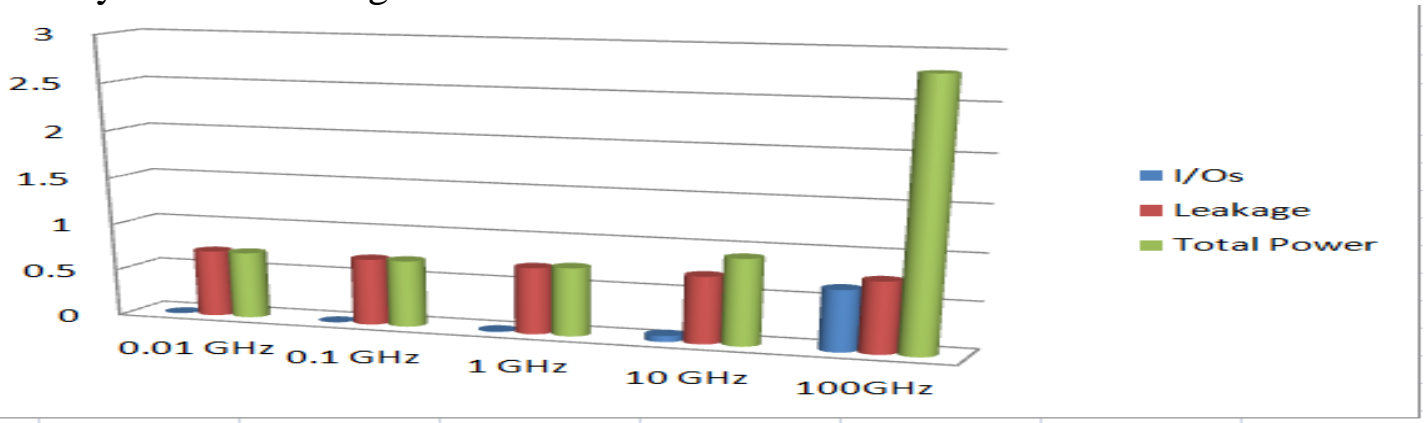

Figure1: Graphical Representation of I/O, Leakage and Total Power for LVCMOS12

\section{B. Result for LVCMOS15}

Table 2: Readings of I/Os, Leakage and Total Power for LVCMOS15 at frequency range from $0.01 \mathrm{GHz}$ to $100 \mathrm{GHz}$

\begin{tabular}{|l|l|l|l|l|l|}
\hline Frequency GHz & 0.01 & 0.1 & 1 & 10 & 100 \\
\hline I/Os & 0.000 & 0.710 & 0.007 & 0.072 & 0.723 \\
\hline Leakage & 0.711 & 0.711 & 0.711 & 0.716 & 0.762 \\
\hline Total Power & 0.711 & 0.713 & 0.732 & 0.928 & 2.881 \\
\hline
\end{tabular}

Readings noted in Table 2 are analyzed and decrease in power consumption is noticed. The data can be analysed clearly from Fig. 2 for LVCMOS15 and Percentage decrease are as follows. Decrease in I/O is $90.4 \%, 99.03 \%, 99.86 \%$ and $100 \%$ when frequency is reduced from $100 \mathrm{GHz}$ to $10 \mathrm{GHz}, 1 \mathrm{GHz}, 0.1 \mathrm{GHz}$, and $0.01 \mathrm{GHz}$ respectively. Decrease in Leakage is $6.03 \%, 6.69 \%, 6.69 \%$ and $6.69 \%$ when frequency is reduced from $100 \mathrm{GHz}$ to $10 \mathrm{GHz}, 1 \mathrm{GHz}$, 
Gyancity Journal of Electronics and Computer Science,

Vol.1, No.1, pp.25-28, September 2016

ISSN: 2446-2918 DOI: 10.21058/gjecs.2016.11005

$0.1 \mathrm{GHz}$, and $0.01 \mathrm{GHz}$ respectively. Decrease in Total Power is $67.78 \%, 74.59 \%, 75.25 \%$ and $75.32 \%$ when frequency is reduced from $100 \mathrm{GHz}$ to $10 \mathrm{GHz}, 1 \mathrm{GHz}, 0.1 \mathrm{GHz}$, and $0.01 \mathrm{GHz}$ respectively as shown in Fig. 2.

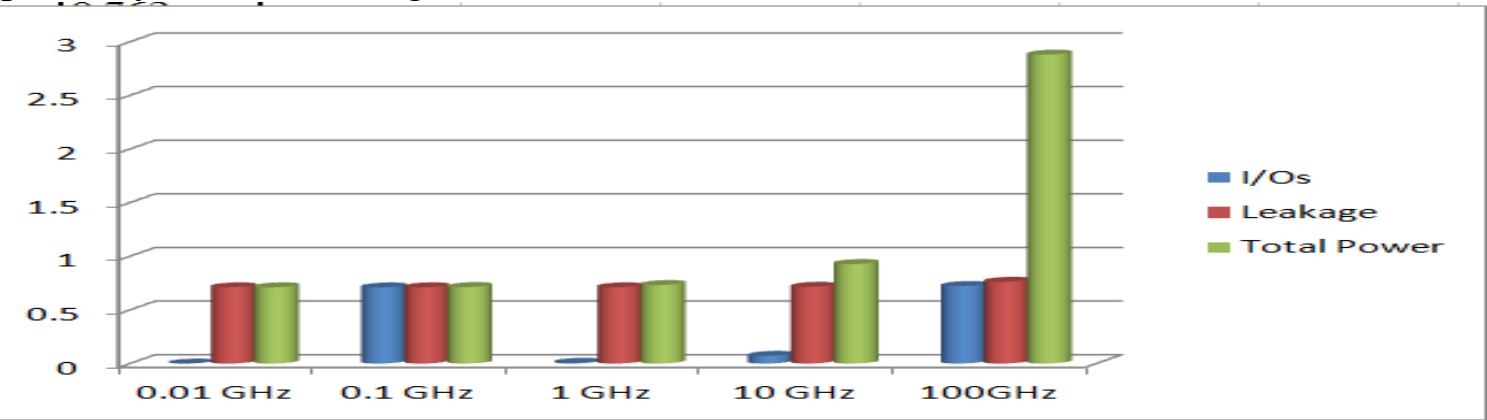

Figure2: Graphical Representation of I/O, Leakage and Total Power for LVCMOS15

\section{Result for LVCMOS18}

Table 3 : Readings of I/Os, Leakage and Total Power for LVCMOS18 at frequency range from $0.01 \mathrm{GHz}$ to

\begin{tabular}{|l|l|l|l|l|l|}
\hline Frequency GHz & 0.01 & 0.1 & 1 & 10 & 100 \\
\hline I/Os & 0.000 & 0.001 & 0.08 & 0.079 & 0.786 \\
\hline Leakage & 0.711 & 0.711 & 0.711 & 0.716 & 0.764 \\
\hline Total Power & 0.711 & 0.713 & 0.733 & 0.935 & 2.947 \\
\hline
\end{tabular}

Readings noted in Table 3 are analyzed and decrease in power consumption is noticed. The data can be analyzed clearly from Figure 3 for LVCMOS18 and Percentage decrease are as follows. Decrease in I/O is $89.94 \%, 98.98 \%, 99.87 \%$ and $100 \%$ when frequency is reduced from $100 \mathrm{GHz}$ to $10 \mathrm{GHz}, 1 \mathrm{GHz}, 0.1 \mathrm{GHz}, 0.01 \mathrm{GHz}$ respectively. Decrease in Leakage is $6.28 \%, 6.93 \%, 6.93 \%$ and $6.93 \%$ when frequency is reduced from $100 \mathrm{GHz}$ to $10 \mathrm{GHz}, 1 \mathrm{GHz}$, $0.1 \mathrm{GHz}, 0.01 \mathrm{GHz}$ respectively. Decrease in Total Power is $68.27 \%, 75.12 \%, 75.80 \%$ and $75.97 \%$ when frequency is reduced from $100 \mathrm{GHz}$ to $10 \mathrm{GHz}, 1 \mathrm{GHz}, 0.1 \mathrm{GHz}, 0.01 \mathrm{GHz}$ respectively as shown in Fig. 3.

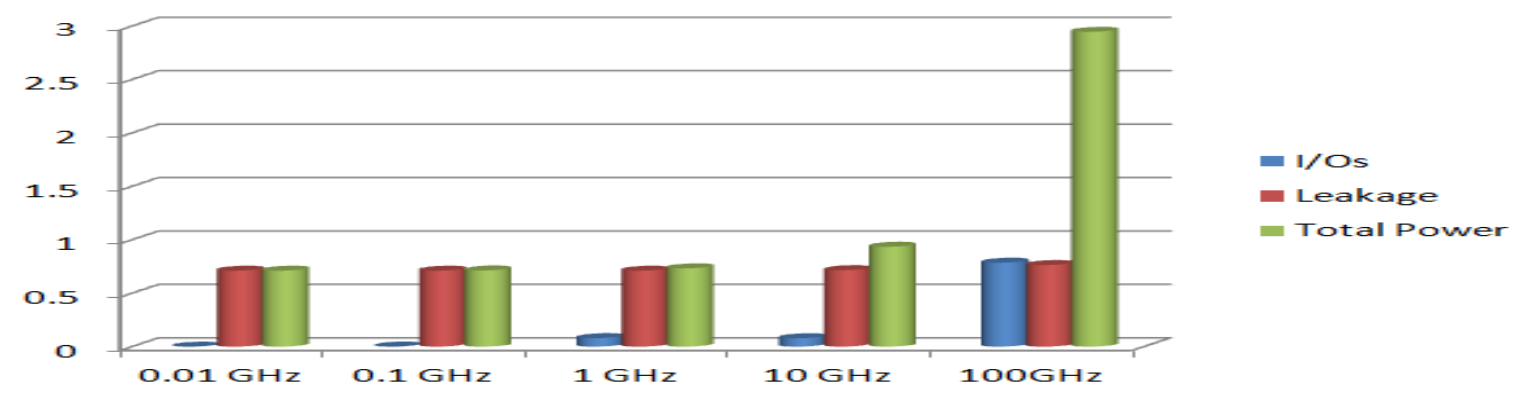

Figure3: Graphical Representation of I/O, Leakage and Total Power for LVCMOS18

\section{Result for LVCMOS25}

Table 4: Readings of I/Os, Leakage and Total Power for LVCMOS25 at frequency range from $0.01 \mathrm{GHz}$ to $100 \mathrm{GHz}$

\begin{tabular}{|l|l|l|l|l|l|}
\hline Frequency GHz & 0.01 & 0.1 & 1 & 10 & 100 \\
\hline I/Os & 0.001 & 0.000 & 0.010 & 0.095 & 0.953 \\
\hline Leakage & 0.712 & 0.712 & 0.712 & 0.717 & 0.769 \\
\hline Total Power & 0.714 & 0.736 & 0.736 & 0.953 & 3.119 \\
\hline
\end{tabular}


Readings noted in Table4 are analyzed and decrease in power consumption is noticed. The data can be analyzed clearly from Figure3 for LVCMOS25 and Percentage decrease are as follows. Decrease in I/O is $90.4 \%, 98.92 \%, 99.84 \%$ and $100 \%$ when frequency is reduced from $100 \mathrm{GHz}$ to $10 \mathrm{GHz}, 1 \mathrm{GHz}, 0.1 \mathrm{GHz}$, and $0.01 \mathrm{GHz}$ respectively. Decrease in Leakage is $5.92 \%, 6.44 \%, 6.57 \%$ and $6.57 \%$ when frequency is reduced from $100 \mathrm{GHz}$ to $10 \mathrm{GHz}, 1 \mathrm{GHz}$, $0.1 \mathrm{GHz}$, and $0.01 \mathrm{GHz}$ respectively. Decrease in Total Power is $67.2 \%, 73.97 \%, 74.65 \%$ and $74.72 \%$ when frequency is reduced from $100 \mathrm{GHz}$ to $10 \mathrm{GHz}, 1 \mathrm{GHz}, 0.1 \mathrm{GHz}$, and $0.01 \mathrm{GHz}$ respectively as shown in Fig. 4.

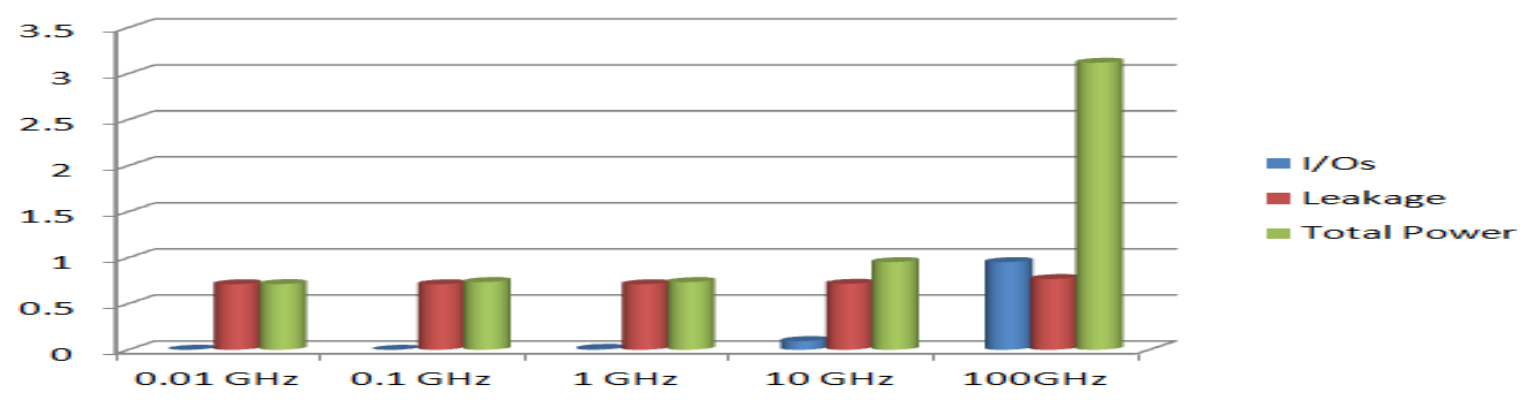

Fig. 4: Graphical Representation of I/O, Leakage and Total Power for LVCMOS25

\section{CONCLUSION}

Work is done successfully and results are analyzed for percentage reductions in leakage, IOs and total amount of power. The maximum decrease in power for LVCMOS12 is $74.72 \%$, for LVCMOS15 is $75.32 \%$, for LVCMOS18 is 75.975 and for LVCMOS25 is $77.17 \%$. From these results it can be concluded that among LVCMOS12, LVCMOS15, LVCMOS18 and LVCMOS25, default standard i.e. LVCMOS 25 shows maximum amount of decrease in power. Percentage decrease in power also increases as we increase the frequency for all the standards.

\section{FUTURESCOPE}

These solar charge sensors will play very important role if used for solar batteries and can conserve large amount of energy as the life span of charging cells will be increased. Number of modifications can be done for reliable design .Voltage Scaling or capacitance scaling can also be used for further work. Time analyses can be done in future. The same can be performed for no of standards present like HSTL, LVDCI [5], SSTL and PCI3_33 etc. Many Field programmable Gate arrays like Virtex-7, Kintex-7, Artix-7 [6] can be used for more study and results for efficient system.

\section{REFERENCES}

[1] A. Singla, , A. Kaur and B. Pandey, ,LVCMOS based energy efficient solar charge sensor design on FPGA. In Power Electronics 6th India International Conference on (pp. 1-5). IEEE, 2014

[2] Semeraro, Greg, et al. "Energy-efficient processor design using multiple clock domains with dynamic voltage and frequency scaling." High-Performance Computer. IEEE, 2002.

[3] Semeraro, Greg, et al. "Dynamic frequency and voltage control for a multiple clock domain microarchitecture." Microarchitecture, Proceedings. 35th Annual ACM International Symposium on. IEEE, 2002.

[4] Simjee, Farhan ., and Pai H. Chou. "Efficient charging of supercapacitors for extended lifetime of wireless sensor nodes." IEEE Transactions on power electronics 23.3 (2008): 1526-1536.

[5] B. Pandey and R.Kumar. "Low voltage DCI based low power VLSI circuit implementation on FPGA." Information \& Communication Technologies, IEEE, 2013.

[6] Przybus, Brent. "Xilinx redefines power, performance, and design productivity with three new $28 \mathrm{~nm}$ fpga families: Virtex-7, kintex-7, and artix-7 devices." Xilinx White Paper, 2010. 